\title{
Histoire et humanités numériques : nouveaux terrains de dialogue entre les archives et la recherche ${ }^{1}$
}

\author{
Frédéric CLAVERT \\ Johanna DANIEL \\ Hélène FLECKINGER \\ Martin GRANDJEAN \\ Fatiha IDMHAND
}

\section{Introduction}

Comme dans toute discipline au contact de nouvelles technologies, on assiste au sein des sciences historiques, et plus largement au sein des sciences humaines et sociales, à un questionnement sur l'adoption de nouvelles pratiques de recherche. Alors que les outils numériques offrent des perspectives d'analyse renouvelées - des méthodes quantitatives complexes aux bases de données multimédia en passant par la visualisation - ceux-ci modifient également les canaux de diffusion et de popularisation du patrimoine, quand ils ne deviennent pas eux-mêmes de nouveaux objets d'étude, à l'image du Web ou des médias sociaux. Sous le titre d'«humanités numériques» se rassemble depuis quelques années une communauté d'individus et d'institutions qui se préoccupent de ces transformations et s'approprient petit à petit un outillage numérique transdisciplinaire. Sur le terrain, loin d'une révolution annoncée

\footnotetext{
${ }^{1}$ Cet article est le compte-rendu de la table ronde " La recherche en histoire à l'ère numérique » organisée par Humanistica, l'association francophone des humanités numériques (www.humanisti.ca), lors du Forum des archivistes 2016 de l'AAF.
} 
avec grandiloquence, c'est par l'expérimentation et la collaboration, pas à pas, que ces nouvelles modalités se mettent en place.

Cet article fait échanger quatre personnalités dont la diversité des parcours et des expériences n'a d'égale que la variété des champs disciplinaires touchés par le phénomène des « humanités numériques ».

Fatiha Idmhand est professeur à l'Université de Poitiers, spécialiste d'études littéraires (hispaniques) et de génétique des textes. Dans le champ des humanités numériques, elle est impliquée dans l'édition électronique des manuscrits, dans leur exploitation numérique et, plus largement, dans l'informatisation de la recherche et des études en lettres.

Spécialiste en stratégie de communication et de valorisation du patrimoine par les technologies numériques, Johanna Daniel est diplômée de l'École du Louvre et de l'École des chartes. Blogueuse prolifique, elle est suivie par une large communauté de passionnés de muséologie, d'estampes et de curiosités culturelles.

Historienne du cinéma et de la vidéo, Hélène Fleckinger est maitresse de conférences à l'Université Paris 8 . Ses travaux portent notamment sur l'apport des technologies numériques à une contextualisation «en rhizomes» des archives audiovisuelles et à leur valorisation scientifique, pédagogique et patrimoniale (comme dans le cas de la plateforme «Bobines féministes », créée avec Nadja Ringart au sein du Labex Arts-H2H).

Frédéric Clavert, maître assistant en histoire contemporaine à l'Université de Lausanne, est un spécialiste de l'épistémologie de l'histoire à l'ère numérique. Il mène des recherches sur les commémorations de la Première Guerre mondiale sur Twitter et de manière générale sur notre rapport au passé dans les médias sociaux.

La discussion est animée par Martin Grandjean, chercheur en histoire contemporaine à l'Université de Lausanne et porte-parole d'Humanistica, l'association francophone des humanités numériques. Cet échange se structure selon deux axes principaux : les conséquences du changement d'outils et les questionnements sur l'enseignement des nouvelles technologies, il se conclut par des points de vue personnels à propos de la définition de ce nouveau champ d'études. 


\section{Changer d'outils, changer d'échelle}

Martin GRANDJEAN : Aujourd'bui, la disponibilité d'outils permettant de gérer, d'analyser et de visualiser de grands corpus de données place souvent le chercheur face à la tentation d'étendre son objet de recherche et d'en faire une lecture distante'. En quoi l'irruption de ces nouveaux outils et la massification des corpus qui l'accompagne modifientt-elles les pratiques du chercheur?

Frédéric CLAVERT : La lecture distante des sources primaires n'est pas un besoin récent des historiens, même si elle n'a pas été formulée en tant que telle avant certains travaux de Franco Moretti. Lors d'une recherche, nous avons tous, à un moment, besoin de prendre une forme de distance vis-à-vis de nos sources, afin d'en retirer une logique et une signification globales. Sans invalider la lecture proche des documents, fondamentale, il faut rappeler qu'un document pris seul n'a tout simplement pas le même sens que s'il est analysé en relations avec d'autres archives. Ce que les grandes écoles historiographiques du $\mathrm{XX}^{\mathrm{e}}$ siècle - en premier lieu les Annales - puis, plus récemment, la mise en données des archives ont changé, c'est une sorte d'extension du domaine de l'histoire : non seulement nous étudions des sujets qui n'étaient pas objet d'histoire auparavant, mais en plus nous pouvons accéder à nos sources plus facilement et les agréger dans des corpus massifs. Le besoin de lecture distante change alors, nous forçant à faire appel à des outils informatiques comme la fouille de texte par exemple.

Cette lecture distante de corpus massifs oblige l'historien à faire attention aux conséquences de la médiation computationnelle introduite par ces outils. Ces conséquences sont diverses - le risque d'arracher les sources primaires à leur contexte d'origine en est un exemple. L'historien doit alors faire très attention à son dispositif technique : les allers-retours entre lecture distante d'un corpus et lecture proche d'un ou de plusieurs documents en particulier doivent être possibles.

Si l'historien doit être attentif à ces éléments de méthodologie, l'accès et l'analyse de corpus massifs doivent être vus aussi sous leurs angles positifs. On peut aujourd'hui traiter des sujets anciens sous un angle nouveau (notamment en accroissant les sources utilisées) ou des sujets qui, faute d'outils, n'étaient pas auparavant appréhendables « humainement».

\footnotetext{
${ }^{1}$ MORETTI (Franco), Distant Reading, Verso Books, 2013, 257 p.
} 
Fatiha IDMHAND : Du côté des sciences du texte, la lecture distante est inscrite dans son " horizon de lecture » mais les moyens à déployer pour la réaliser semble encore peu accessibles, notamment parce que l'exégèse des textes pratique une lecture de proximité, une lecture fine visant la plus grande exhaustivité. Pour cela, l'historien travaille avec un corpus d'oeuvres limité qu'il a organisé autour d'une thématique. C'est à partir de cette lecture attentive à tous les détails d'un texte ou de quelques textes qu'il construit un discours scientifique. L'accès à une plus grande masse de textes permettrait d'étayer et de systématiser les hypothèses qui sont élaborées à partir de ces quelques sources, mais le changement impliqué par ce mode de lecture est une révolution signifiant à la fois la fin d'une forme de lecture savante dirigée, en amont, par l'humain. La perspective est évidemment à la fois alléchante et effrayante : l'idée d'éprouver les théories développées autour de quelques écrits à une échelle supérieure et planétaire laisse évidemment présager de grandes possibilités pour la recherche. Or il faut à la fois former l'humain mais également éduquer les machines. Dans le même temps, il s'agit de repenser, d'inventer les fondements pratiques et théoriques de ces " nouvelles lectures savantes $»$.

Nous n'y sommes pas encore! Notamment parce que les «humanistes numériques » des sciences du texte sont très occupés à transformer leurs objets d'études et de recherches en objets numériques (mixtes le plus souvent, papier et numérique), à les éditer enrichis de savoirs critiques dans des formats interopérables et à publier leurs résultats de façon à poser les bases de nouveaux cadres théoriques.

Martin GRANDJEAN : Or, dans la pratique, la faisabilité d'une telle "lecture distante »n'est-elle pas très largement conditionnée par l'accessibilité réelle de ces corpus?

Johanna DANIEL : Si nous disposons aujourd'hui d'outils et de méthodologies permettant l'exploration et la lecture de corpus de données importants, il demeure encore d'importants obstacles. Prenons l'exemple des sources numérisées et disponibles dans les bibliothèques numériques, sur les sites d'archives en ligne ou dans les bases de données patrimoniales: les documents sont disponibles à la consultation, parfois au téléchargement, mais seulement item par item. Or, le chercheur peut avoir besoin de télécharger en masse les données pour les exploiter dans ses propres outils de fouille de texte, 
d'analyse d'image ou de transcription. Ces usages-là, ces outils-là n'ont pas toujours été envisagés par les institutions patrimoniales, qui mettent un tas d'obstacles, justifiés ou non, interdisant l'enregistrement des images, restreignant le téléchargement au format PDF, empêchant l'export massif de résultats de recherches, etc. Ces freins obligent les chercheurs à bidouiller (requêtes en ligne de commande, etc.), voir à « hacker» les bases de données pour arriver à leurs fins. N'y aurait-il pas là de nouvelles pistes de travail et de collaboration pour les institutions patrimoniales, afin de favoriser ces usages nouveaux, ces réutilisations au service de la recherche?

Hélène FLECKINGER : Dans le domaine des archives audiovisuelles, la massification des sources numérisées et nativement numériques est un phénomène ambivalent, à la fois enthousiasmant et étourdissant. D'un côté, elle offre de nouvelles perspectives de recherche, notamment en histoire contemporaine, en renouvelant les regards et en apportant une charge sensible singulière. De l'autre, nous sommes submergés par des volumes sans cesse croissants - l'Institut national de l'audiovisuel (Ina) dispose ainsi de plus de 15 millions d'heures d'enregistrement - et nous ne ne savons pas toujours comment appréhender des fonds qui ne sont pas systématiquement documentés avec précision. Si un historien a l'habitude de travailler avec des traces lacunaires, si la prise en compte des manques fait partie intégrante de son processus de travail, la surabondance de données vient paradoxalement troubler sa méthodologie. Que faire face à un trop-plein d'images et de sons, souvent privés d'informations sur leur contexte de production et de diffusion? Ou une illusion de trop-plein, car la profusion de certaines sources n'est pas contradictoire avec l'absence de fonds relevant de points de vue différents, voire divergents, et peut justement augmenter les risques de biais dans l'écriture de l'histoire.

Une difficulté majeure me semble bien résider dans la nécessité de jouer sur des échelles complémentaires, mais parfois malaisées à combiner : lecture distante et rapprochée, prise en considération de vastes ensembles et analyse fine de fragments. Mais nous manquons encore d'outils performants pour traiter les archives audiovisuelles dans leur complexité et leur granularité. La fouille de données audiovisuelles pose des problèmes non résolus et les outils d'annotation intra-filmique, si certains sont anciens, ne permettent toujours pas la redocumentarisation de corpus audiovisuels massifs, contrairement à ce qui se pratique dans le domaine des images fixes. Du point de vue de l'histoire du temps présent, c'est pourtant là un enjeu essentiel: utiliser les outils 
numériques de façon collaborative entre chercheurs certes, mais aussi en associant les producteurs d'archives audiovisuelles, les acteurs et les témoins au travail d'identification et d'analyse, afin d'enrichir les connaissances.

Martin GRANDJEAN : Le changement d'échelle ne se vit pas seulement au niveau de la recherche mais également dans le changement de médium de communication de celle-ci. Internet, en plus d'être aujourd'bui devenu une source à part entière, est également un moyen de diffusion de contenus : comment les blogs et les réseaux sociaux peuvent-ils être mis à profit dans le cadre d'un partage de connaissances ou de ressources?

Johanna DANIEL : Au-delà de l'échange entre professionnels de la recherche, Internet a permis de créer de nouvelles formes de médiation de la science et du patrimoine à destination du grand public. Sur des plateformes comme Hypothèses, on trouve essentiellement des blogs de chercheurs ou jeunes chercheurs qui s'adressent à des collègues. Mais d'autres formes existent: certains doctorants s'essayent à des formes de médiation plus populaires, sur YouTube par exemple. La doctorante Manon Bril (université Jean-Jaurès, Toulouse) a ouvert la chaîne "c'est une autre histoire » consacrée à la représentation des mythes dans l'art. Son succès fulgurant (48000 abonnés en un an) a conduit le magazine numérique Mondes sociaux (édité par le labex SMS) à lui commander une émission Avides de recherche dans laquelle Manon Bril explique des travaux de recherche en Sciences humaines et sociales à un public large.

Pour ma part, c'est certainement mon goût pour la médiation qui m'a poussée à créer, il y a cinq ans, le blog Orion en aéroplane, consacré au patrimoine. Espace d'écriture personnel, il a évolué à mesure de mon cursus : j’y ai partagé mes premières expériences de la recherche comme mes coups de coeur dans les expositions. Cette expérience du blogging m'a permis de forger des compétences professionnelles en médiation numérique, qui m’ont ensuite menée à contribuer à des projets numériques auprès d'institutions culturelles.

Qu'il s'agisse de blog, de chaîne YouTube, de comptes sur les réseaux sociaux, ces pratiques nouvelles induisent également des tensions nouvelles : ce sont des espaces hybrides, où l'on parle autant de soi que de sa recherche. Vie privée et image publique se mêlent, si bien qu'il n'est pas toujours facile de trouver le bon ton, la bonne place pour chaque chose. Ce sont de nouveaux défis auquels il faut apprendre à répondre. 
Frédéric CLAVERT : Je ne peux ici qu'évoquer ma seule expérience. Bloguer a facilité mon écriture, m'a permis d'ouvrir un espace où, contrairement à un livre, un article ou même un enseignement, j'ai le droit à l'erreur. Fréquenter les réseaux sociaux m'a permis d'enrichir mes relations avec de nombreux collègues et, notamment, avec des collègues chercheurs mais non-historiens mais aussi des collègues archivistes, conservateurs des bibliothèques ou encore ingénieurs. Car l'une des particularités de la recherche en histoire à l'ère numérique me semble être la transdisciplinarité et la revitalisation, d'une certaine manière, des sciences auxiliaires de l'histoire.

Martin GRANDJEAN : Et au-delà des blogs et des réseaux sociaux, Internet accélère et démocratise la diffusion des contenus scientifiques, voire permet l'émergence de nouvelles formes de publication.

Fatiha IDMHAND : Indéniablement, Internet a d'abord facilité l'accès à la recherche. Les publications de certains domaines des Sciences humaines et sociales ont toujours eu beaucoup de mal à circuler, à la fois par manque de diffusion des éditeurs, mais également en raison de l'échelle des éditions, souvent locales, assez exclusives et par conséquent impossibles à trouver. Les grands portails de revues, les pages personnelles, les sites des centres de recherches, les blogs nous ont permis d'accéder à toute la connaissance internationale. L'état de l'art est donc facilité par Internet et il devient difficile d'ignorer ce qui a été publié et diffusé en ligne. Il est donc important que les travaux et les résultats des recherches circulent pour apparaitre dans les états de l'art et pour permettre, dans le même temps, d'actualiser la recherche. Les réseaux sociaux contribuent à cet état de l'art et à la prise de contact : ils permettent de suivre en temps réel les travaux des équipes, de diffuser, de nouer des dialogues et de réaliser la discussio, l'examen attentif, contradictoire.

Hélène FLECKINGER : Internet a permis l'émergence de nouveaux projets éditoriaux, hybrides, utilisant avec profit les avantages du numérique : accès facilité à des documents parfois dispersés, intégration de ressources multimédia, lecture enrichie grâce à des liens hypertextes, inclusion de pratiques collaboratives, etc. Pour la valorisation scientifique, une plateforme 
comme Hypothèses est particulièrement pertinente : un carnet de recherche peut jouer le rôle de journal de bord d'une recherche individuelle ou collective en cours, en mettant à disposition des sources et en diffusant des analyses. Ce type de blog est un puissant outil de mise en réseau de recherches vivantes.

Mais on observe aussi actuellement une multiplication des plateformes de médiation des mémoires en ligne, c'est-à-dire des oeuvres numériques reposant sur la mise en récit du passé, qui s'imposent à la fois comme des lieux d'analyse et de partage des connaissances. Désormais, le concept de lieux de mémoire défini par Pierre Nora se décline en numérique, et ce phénomène majeur est évoqué par Claire Scopsi dans son carnet de recherche "Passerelle de mémoire ${ }^{1}$, sur les enjeux et méthodes des collectes de mémoires à l'ère numérique. Fondée sur une base de données évolutive réunissant des matériaux hétérogènes (films, documents sonores, images fixes, archives écrites, etc.), la plateforme «Bobines féministes $»^{2}$, que je co-dirige avec Nadja Ringart (accessible en ligne pour tout public début 2017), s'inscrit dans cette perspective. Destinée à la valorisation scientifique, pédagogique et patrimoniale de ressources numériques sur le Mouvement de libération des femmes dans les années 1970 en France, qu'elle entend recontextualiser et croiser, elle répond à la volonté d'écrire de manière polyphonique une histoire collective et, pour cela inclura un volet participatif (collecte de documents numérisés, annotations, etc.). La dimension inventive de ces nouvelles publications numériques ne doit pas non plus être négligée : elles incarnent de nouvelles fabriques de connaissances mais deviennent aussi des lieux de création en soi.

\section{Enseigner le numérique et s'y former soi-même}

Martin GRANDJEAN : La question de la formation « au numérique 》, pour autant que celui-ci puisse être substantivée, est souvent considérée comme un point de détail qui découlerait logiquement des pratiques d'une poignée de pionniers ouvrant la voie aux générations futures. Mais c'est une préoccupation majeure de celles et ceux qui, au-delà de leur activité de recherche, sont confrontés à la mise à jour des cursus universitaires. Qu'enseigner? "Le numérique » comme discipline propédentique à part entière ou "le numérique » insufflé dans les disciplines déjà existantes?

\footnotetext{
1 http://passerelle.hypotheses.org

2 http:/ / vitrines2014.labex-arts-h2h.fr/content/bobines-féministes

3 MOATTI (Alexandre), "Le numérique, adjectif substantivé », Le Débat, 3, n 170, 2012, p. 133-137.
} 
Hélène FLECKINGER : Ma vue est très partielle dans le domaine et j'ai tendance à adopter le prisme des disciplines artistiques. Même dans des formations articulant théorie et pratique de la création, on constate souvent une étrange absence de prise en compte des conditions matérielles de production : bien qu'étroitement imbriqués avec les questions formelles, les moyens techniques - en général - sont trop souvent oubliés dans l'analyse des œuvres. Dans les études cinématographiques, contrairement aux arts plastiques, le rapport au numérique se révèle ambigu et se limite à l'appropiation artistique de technologies numériques lors du tournage puis du montage, virtuel mais linéaire, car les approches délinéarisées (de type narrations connectées, Webdocumentaires, transmédia) sont très mal considérées. Bien qu'ils soient de puissants instruments d'analyse (de «démontage ») de films, les outils d'annotation intra-filmique sont par ailleurs sous-utilisés. À mon modeste niveau, je m'attache donc à introduire les questions liées au numérique dans tous mes enseignements et selon ses multiples déclinaisons, et à faire en sorte que ces réflexions essaiment grâce aux étudiants, en effet très demandeurs.

Si on perçoit la nécessité de les former à une certaine culture numérique, il y a probablement aussi une pédagogie à entreprendre auprès de nos propres collègues universitaires, un effort à mener d'abord pour les sensibiliser à l'intérêt d'inclure des pratiques et usages numériques au sein de leur discipline, puis pour les emmener sur des chemins plus transversaux. Car les approches numériques exigent un déplacement qui peut être très inconfortable et certains chercheurs ressentent la transdisciplinarité comme une menace pour leurs habitus scientifiques et pédagogiques. S’orienter vers les humanités numériques exige de fait de se former soi-même, or expérimenter sur ce terrain n'est pas du goût de tous.

Fatiha IDMHAND : Au-delà de l'enseignement du numérique qui recouvre effectivement de très nombreux aspects qui vont du code informatique à l'approche transversale d'un objet d'étude et de recherches, c'est la compréhension d'un nouveau monde qui est actuellement au cœur des formations. Or les formations sont très en retard et, actuellement, nous travaillons au coup par coup et au cas par cas : les formateurs se forment presque en même temps que ceux qu'ils doivent former à des technologies qui évoluent en permanence. La formation du formateur (qu'il soit enseignantchercheur ou autre) est également mise à rude épreuve. 
Bien sûr, c'est avant l'arrivée à l'université qu'il faudrait former à la «culture numérique » et aux notions et langages de l'informatique afin que l'université puisse permettre aux étudiants d'affiner et de se spécialiser. En attendant, l'enseignement supérieur peut/devrait/doit intégrer des pratiques numériques qui permettront déjà aux étudiants de mieux comprendre leur nouveau monde informatisé.

Martin GRANDJEAN : Face à ces constats, faut-il renforcer l'enseignement de l'informatique?

Johanna DANIEL : Au-delà du fantasme emblématique d'enseigner le code, il y a une urgence à former les étudiants aux bases de l'informatique. En master, nous voyons arriver des étudiants qui ne maitrisent aucun outil de bureautique, pas même le traitement de texte. Alors que les écoles et universités voudraient nous voir former les étudiants à la création de bases de données et de sites Web, nous avons du mal à convaincre qu'il faut consacrer du temps et de l'argent à l'acquisition de compétences aussi simples que la bureautique.

Le fossé générationnel s'exprime aussi là : les décideurs, plus âgés, pensent que la génération $\mathrm{Y}$, parce qu'elle est à l'aise avec un smartphone ou Facebook, maitrise parfaitement tous les outils numériques. Or, mettre en page correctement un document, effectuer une recherche dans une base de données sont des choses qui exigent de la rigueur et de la méthode qu'en général seuls les étudiants qui ont une appétence pour cela ont acquis. EEtre digital native ne fait pas des étudiants des génies de l'informatique. Par ailleurs, on observe une certaine méfiance vis-à-vis de l'ordinateur chez certains étudiants, qui le perçoivent comme une boîte noire impénétrable, capable d'« avaler» du jour au lendemain toutes leurs données. Il faut donc prendre le temps d'enseigner la culture numérique, et ce dès la licence.

Théoriquement, le certificat de compétences informatiques et Internet, délivré par les universités au cours du cursus LMD, répond à ce besoin. Malheureusement, beaucoup d'étudiants considèrent cet enseignement comme secondaire, d'autant qu'un échec à l'examen ne menace pas la poursuite de leurs cursus.

Frédéric CLAVERT : La formation informatique, la formation au numérique est un vaste sujet, traité régulièrement, en France par exemple, depuis au moins 
trente ans et toujours pas vraiment résolue ${ }^{1}$. À partir du master, je pense qu'aujourd'hui, la formation est plutôt correcte. Il y a des masters emblématiques, comme celui de l'École des chartes et il existe des masters dans le monde francophone qui sont en train d'ouvrir, comme à Lausanne. Le problème me semble se situer avant le master : rares sont les universités qui, comme à Lille par exemple, forment leurs étudiants au numérique dès le premier semestre de licence.

Une fois que l'on a posé le principe selon lequel la formation au numérique doit commencer le plus tôt possible, encore faut-il définir le contenu de cette formation. Je suis pour une stratégie du pas à pas : commencer par enseigner une sorte de culture numérique, puis spécialiser de plus en plus les enseignements, en les adaptant à chaque formation. La question souvent considérée comme centrale, celle de l'apprentissage du code, me semble dépendre, justement, de la formation dans laquelle il est enseigné. Il est parfois plus pertinent d'apprendre aux historiens à parler avec des ingénieurs, notamment, pour mettre en place des projets numériques : pour cela, il faut un langage commun, donc une culture commune.

Martin GRANDJEAN : Dans une telle période d'évolution technologique, les nowvelles expertises développées dans le cadre de ces formations peuvent se trowver en décalage avec les attentes d'un marché du travail académique encore très traditionnel. Comment valoriser ces compétences face à des cabiers des charges qui évoluent peu? Au-delà d'une thèse de doctorat, y a-t-il un avenir professionnel pour ces profils très spécifiques?

Fatiha IDMHAND : La question sous-jacente est celle des métiers de demain. Nous formons à des métiers qui n'existent pas encore, beaucoup de nos étudiants vont inventer leurs métiers et pour les y aider, nous devons nousmêmes redoubler d'efforts, d'imagination et de polyvalence. C'est une situation très différente de celle d'il y a encore dix ans : beaucoup de métiers ont disparu, d'autres ont évolué, certains sont apparus.

Frédéric CLAVERT : La question des débouchés ne se pose-t-elle vraiment que pour ces profils spécifiques?

1 On rappellera par exemple: GENET (Jean-Philippe), «La formation informatique des historiens en France: une urgence », Mémoire vive, 9, 1993 et, près de vingt ans après, RUIZ (Émilien) et HEIMBURGER (Franziska), «Faire de l'histoire à l'ère numérique : retours d'expériences ", Revue d'bistoire moderne et contemporaine, $\mathrm{n}^{\circ}$ 58-4bis (5), 2011, p. 70-89. 
Johanna DANIEL : Il y a une forte demande sur le marché de ces profils spécifiques, polyvalents, capables de développer des projets numériques dans des structures liées aux sciences humaines (bibliothèques, musées, archives, Maisons des sciences de l'homme), comme en témoignent les très nombreuses offres postées à chaque rentrée. Le taux d'insertion professionnelle du master Technologies numériques appliquées à l'Histoire témoigne également de cette demande qui va croissant : la plupart des étudiants trouvent un emploi dans les mois qui suivent le diplôme, quand ce n'est pas avant.

Le problème ne relève pas tant de l'existence d'une demande que de la reconnaissance réelle de ces nouvelles compétences. Les salaires demeurent très bas, or les candidats sont souvent titulaires de deux masters, l'un en sciences humaines et l'autre lié aux nouvelles technologies. L'absence du titre de docteur peut être un frein au recrutement, alors même que ces personnes ont toutes les compétences opérationnelles exigées sur les fiches de poste.

D'autre part, si les institutions ressentent de plus en plus le besoin d'avoir des agents au fait des nouvelles technologies, les structures ne sont pas toujours adaptées aux nouvelles manières de travailler.

Ces nouveaux profils sont surtout des chefs de projets, capables de coordonner une équipe technique au service d'un projet scientifique. Or, dans les faits, si les institutions sont prêtes à recruter ces nouveaux profils, elles ont rarement les moyens pour payer également une équipe technique. On demande alors à ces ingénieurs d'études de porter toutes les casquettes: chef de projet, informaticien, webdesigner, webmaster, etc. Ce qui n'est pas bon : ces profils sont polyvalents mais pas magiciens.

Hélène FLECKINGER : Je ne peux pas m'empêcher de penser au slogan de Mai 68 «Cours, camarade, le vieux monde est derrière toi » comme métaphore de la situation que vivent en effet certains jeunes professionnels : une situation de décalage parfois difficile dans un contexte de précarité généralisée, mais qui sait aussi ménager des ouvertures professionnelles grisantes. Si leur savoir-faire est un atout, il n'est pas seulement technique : derrière ces profils se trouvent des compétences nombreuses et précieuses, notamment la capacité à dialoguer avec des professionnels issus de corps de métiers très divers, dans le cadre d'un projet numérique précis. 


\section{Conclusion : quelles « humanités numériques » ?}

Martin GRANDJEAN : Le terme d'"humanités numériques》 voit sa fréquence d'utilisation décuplée ces dernières années : entre mot-clé à la mode dans les demandes de subventions et notion âprement discutée dans certains cercles d'initiés, on comprend qu'il s'agit de questionner notre propre relation à ce concept. Est-ce une discipline, une transdiscipline, un champ, une bybridation, une méta-science, un écosystème, une communauté de pratique?

Frédéric CLAVERT : Les définitions des humanités numériques sont innombrables. Le site Web "What is Digital Humanities ${ }^{1}$ qui en recense un très grand nombre fait plutôt penser à un inventaire à la Prévert. Je préfère en rester simplement à la communauté de pratiques. Les événements d'humanités numériques qui m’ont le plus marqué sont les différents THATCamp ${ }^{2}$ auxquels j'ai participé et que j'ai parfois organisés. Toute la philosophie des THATCamps repose sur l'échange de savoir-faire, sur les discussions autour des expériences de chacun, c'est-à-dire sur les pratiques.

Cela ne signifie pas qu'il ne faut pas mener une réflexion sur nos usages numériques. Il me semble important de thématiser, pour reprendre les termes de la question, cette rencontre entre sciences humaines et technologie à partir de l'un de ces deux moments: quand un projet de recherche nécessite de travailler avec d'autres corps de métier parce que les outils numériques nécessités par cette recherche deviennent trop complexes pour l'historien seul ; quand l'usage des outils numériques entraîne des questions épistémologiques importantes.

Fatiha IDMHAND : Les «humanités » deviennent «numériques » quand les chercheurs organisent l'évolution de leurs pratiques, quand ils font appel à de nouvelles méthodes, à des communautés, bref, quand ils sortent de leur isolement traditionnel et qu'ils acceptent de considérer que la révolution numérique affecte également l'ensemble de l'écosystème de la recherche. Les "humanités numériques» resteront des «humanités » mais avec de nouvelles épistémologies, « informatisées ».

\footnotetext{
1 http://whatisdigitalhumanities.com/

2 «The Humanities and Technology Camp» (http:/ / thatcamp.org), une forme de "non-conférence » dont le programme, souvent constitué d'ateliers et de réflexions sur les pratiques numériques en sciences humaines, est mis au point par les participants eux-mêmes. Voir également, pour les trois THATCamp Paris : http//tcp.hypotheses.org.
} 
Martin GRANDJEAN : Et alors qu'il semble que l'on commence toujours par "faire des bumanités numériques sans le savoir ", à quel moment devient-il intéressant de thématiser cette rencontre entre sciences humaines et technologie?

Hélène FLECKINGER : Si j’ignore encore que je me suis lancée dans les humanités numériques, j'admets néanmoins apprécier l'invocation d'une tradition culturelle humaniste et la résonance collective de l'expression. Que l'informatisation généralisée engendre des bouleversements anthropologiques majeurs est une évidence : elle ne peut être sans conséquence sur la recherche, ses enjeux et ses pratiques. Nous devons en prendre acte et plus encore saisir cette occasion de renouveler nos approches épistémologiques. À l'heure où les appels à projets incluent (voire exigent) systématiquement un volet numérique, à nous de refuser tout opportunisme et de défendre une vision rigoureuse et exigeante des humanités numériques, loin de toute instrumentalisation, et que j'espère pour ma part créatrice et réellement ouverte.

Frédéric CLAVERT

Maître-assistant en histoire contemporaine

Université de Lausanne

frederic.clavert@unil.ch

Johanna DANIEL

Consultante indépendante en valorisation du patrimoine par les technologies numériques

http://johannadaniel.fr contact@johannadaniel.fr

Hélène FLECKINGER

Maîtresse de conférences

Université Paris 8 Vincennes - Saint-Denis helene.fleckinger@univ-paris8.fr

Martin GRANDJEAN Chercheur en histoire, porte-parole d'Humanistica

Université de Lausanne martin.grandjean@unil.ch

Fatiha IDMHAND

Professeur des universités

Université de Poitiers Fatiha.idmhand@univ-poitiers.fr 